\title{
ACTORES SOCIALES Y DESARROLLO DE LA LEY DE DEPENDENCIA EN ESPAÑA
}

\author{
SOCIAL ACTORS AND DEVELOPMENT OF LAW OF DEPENDENCY \\ IN SPAIN
}

\author{
Vicente Marbán Gallego vicente.marban@uah.es \\ Universidad de Alcalá. España
}

\begin{abstract}
RESUMEN
La Ley 39/2006 supone un episodio de cambio fundamental en la política social de atención a la dependencia. En el texto se sostienen las hipótesis de que esta Ley es el resultado tanto de una senda acumulativa (path dependency) de factores socioeconómicos e institucionales acaecidos en la política social de atención a la dependencia desde los 80 , como del resultado de intensos procesos de negociación entre los distintos actores sociales implicados mediante el establecimiento de "coaliciones de apoyo" (advocacy coalition) entre ellos. Para contrastar dichas hipótesis, se analiza el papel de los actores sociales en el desarrollo de la Ley y se realiza una valoración tentativa de sus resultados, retos e incertidumbres hasta la fecha. Con tal fin, se han realizado 17 entrevistas en profundidad a actores sociales estratégicos entre octubre de 2007 y julio 2008 partiendo de un cuestionario semiestructurado complementado con el análisis de fuentes secundarias y documentales.
\end{abstract}

\section{Palabras clave}

Cuidados de larga duración; Estado de Bienestar; Ley 39/2006; Política social; Reformas sociales.

\begin{abstract}
Law 39/2006 amounts to an episode of fundamental change in the social policy of long-term care. This article advances the hypotheses that this legislation is the result both of a path dependency on socio-economic and institutional factors that have come about since the 1980s and of intense negotiating processes between the different social stakeholders involved by means of the setting up of advocacy coalitions. To prove these hypotheses, analysis is made of the role of those stakeholders in the development of the legislation, and a tentative assessment is offered of its results, challenges and uncertainties to date. To this end, 17 in-depth interviews were conducted with strategic social stakeholders between October 2007 and July 2008 on the basis of a semi-structured questionnaire and complemented by an analysis of secondary and documentary sources.
\end{abstract}

\section{KEYWORDS}

Law 39/2006; Long-Term Care; Social Policy; Social Reforms; Welfare State. 


\section{BREVE ENCUADRE TEÓRICO, HIPÓTESIS DE TRABAJO Y METODOLOGíA}

Desde los años 1980 hasta la promulgación de la Ley 39/2006 de Promoción de la Autonomía Personal y Atención a las Personas en Situación de Dependencia de 2006 (LAPAD, en adelante) se han ido articulando paulatinamente distintos tipos de medidas y prestaciones orientadas a cubrir, no tanto situaciones de dependencia tal y como se entiende hoy día este concepto, sino situaciones específicas de envejecimiento y de discapacidad.

Con la LAPAD se desarrolla un derecho universal subjetivo para todos los individuos que, independientemente de la edad, acrediten su residencia de forma estable y que presenten uno de los grados de dependencia contemplados en la ley (moderada, severa o gran dependencia) (Art. 26). La valoración del grado de dependencia se realiza mediante la aplicación de un baremo acordado en el Consejo Territorial en base a un cuestionario y a la observación directa por un profesional cualificado de la persona en su entorno habitual.

En cuanto a los tipos de prestaciones, en la ley se da prioridad a los servicios sobre las prestaciones monetarias $y$, de no ser posible la atención por servicios públicos 0 concertados, se asignaría una prestación económica vinculada a un servicio (Art. 17).

La financiación se realiza con cargo a la imposición general y se prevé que la Administración General del Estado financie la cobertura básica común y que las CCAA aporten una cantidad similar. El Gobierno central debe establecer a tal efecto convenios con las CCAA para cofinanciar las prestaciones. El resto sería financiado con el copago de los beneficiarios según su renta y patrimonio.

Para encuadrar teóricamente el episodio de cambio que supone esta ley se adopta el enfoque de la tríada "ideas, instituciones e intereses" donde se considera a las "ideas" como aquellas que los actores tienen para conseguir sus "intereses" y a las "instituciones" como el procedimiento de conflicto, negociación y consenso que estructura el juego de intereses de dichos actores ${ }^{1}$.

En el ámbito de las ideas destacamos la importancia de los actores colectivos en la formación de las diferentes coaliciones que desembocan en una reforma como la que nos atañe (enfoque de los recursos de poder, (power resources approach). Todo ello en un contexto en el que resulta decisivo el legado histórico o "dependencia de la senda" (path dependency) que han supuesto las reformas sociales, inercias institucionales y presiones sociodemográficas acaecidas desde los años ochenta en la política social de atención a la dependencia.

En el terreno de los intereses sería relevante para nuestro análisis el marco de referencia planteado por Sabatier y Jenkins-Smith (1999) de las "coaliciones de apoyo" (advocacy coalition) que avala la existencia de grupos de actores que comparten

\footnotetext{
${ }^{1}$ Para mayor detalle de este enfoque y su capacidad explicativa para el análisis de las reformas de las políticas del bienestar en España sugerimos la lectura del Capítulo primero de Moreno (2009).
} 
creencias y que buscan la consecución de una serie de objetivos comunes para lo cual establecen entre ellos estructuras organizativas y estrechas redes de contacto y cooperación mutua.

A partir de este breve encuadre teórico, se van a contrastar las siguientes hipótesis:

Hipótesis 1: La LAPAD no supone un episodio de cambio o una reforma radical sido una reforma gradual dependiente de una senda (path dependency) acumulativa de factores socioeconómicos e institucionales acaecidos desde los años 80.

Hipótesis 2: Este episodio de cambio es el resultado de la importancia central, que no determinante, de los actores sociales mediante el establecimiento de "coaliciones de apoyo" (advocacy coalition) basadas en estructuras organizativas y estrechas redes de contacto entre ellos.

Con respecto a la metodología, se han realizado 17 entrevistas en profundidad a los actores sociales implicados (sindicatos, patronal, entidades no lucrativas de acción social, partidos políticos, CCAA, expertos) entre octubre de 2007 y julio 2008, siguiendo un cuestionario semiestructurado que se ha complementado con el análisis de fuentes secundarias y documentales. Las entrevistas se han realizado en el marco del proyecto de investigación Nursopob (Nuevos Riesgos Sociales y Trayectorias de las Políticas del Bienestar) del Plan Nacional de I+D+I del Ministerio de Educación y Ciencia.

El cuestionario consta de varios bloques de preguntas. En el primer bloque se abordan cuestiones generales sobre la evolución de la política social de atención a la dependencia. En el segundo bloque se tratan los principales factores de cambio social e institucional relacionados con dicha política social. En tercer lugar, se tratan en profundidad los pormenores del proceso de cambio que supuso la LAPAD, los contenidos, la valoración de dicho cambio y la dinámica de las relaciones entre los actores implicados $y$, finalmente, un bloque relacionado con el futuro de la LAPAD y sus principales retos

Entre los actores sociales se ha entrevistado a los responsables en temas de dependencia en el Congreso de los Diputados de los principales partidos políticos (PSOE, PP), a representantes de los sindicatos (CCOO y UGT), empresarios (CEOE), incluidos los relacionados con la economía social (LARES, AESTE), entidades no lucrativas de acción social con carácter singular (Cáritas y Cruz Roja), de personas mayores (CEOMA) y de personas con discapacidad (FEAPS), expertos, y directores generales de sanidad y de servicios sociales de programas específicos de atención a la dependencia de distintas CCAA (Cataluña y Asturias, entre otras).

Los criterios de selección y la relevancia de los actores entrevistados responden a su implicación en los procesos de negociación de la Ley de Dependencia, en los acuerdos sociales relacionados con esta contingencia y en la elaboración del Libro Blanco, así como por su relevancia como proveedores de servicios lucrativos y no lucrativos relacionados con la dependencia.

El texto queda estructurado de modo que en los dos primeros apartados se contrastarán respectivamente cada una de las hipótesis y en el tercer apartado se realizará un 
análisis tentativo de los resultados, retos e incertidumbres de la ley hasta la fecha que se completará con un apartado final de breves conclusiones y propuestas para la LAPAD.

\section{LA LAPAD, ¿REFORMA ABRUPTA O GRADUAL? REFORMAS SOCIALES, INERCIAS INSTITUCIONALES Y CONDICIONANTES DE CAMBIO EN LA POLITICA SOCIAL DE ATENCIÓN A LA DEPENDENCIA}

El actual debate sobre una atención integral y sociosanitaria de la dependencia y la actual LAPAD no podrían construirse sin la referencia a las diferentes reformas sociales y factores de cambio acaecidos desde hace tres décadas. Todos ellos han ido configurando un mapa en expansión, aunque descoordinado, de prestaciones para personas mayores y personas con discapacidad que han precipitado la aprobación en 2006 del principal episodio de cambio en la política social de atención a la dependencia: la LAPAD.

Entre ellos destacaríamos fundamentalmente los siguientes aspectos:

a) El contexto europeo, reformas sociales e inercias institucionales en la política social de atención a la dependencia. El episodio de cambio que estamos analizando tiene parte de su referente en las políticas de la UE en materia de cuidados de larga duración, las cuales supusieron la dinamización de una discusión incipiente en España donde, hasta finales de los años 90 , el debate general sobre protección social estuvo protagonizado por la viabilidad y reforma del sistema de pensiones. En este sentido, aunque no es el objetivo de este texto analizarlas con detalle, no se puede pasar por alto que las experiencias europeas, en particular las de los países nórdicos y centroeuropeos (Pacolet 1998), supusieron un acicate más en la definición de una política integral de atención a la dependencia en España (Pavolini y Ranci 2008). Tales experiencias se reflejan desde el año 2005 en los Joint Report de la Comisión (European Commission 2009).

En lo que se refiere a las reformas que en España han ido sentando las bases de la actual LAPAD, serían, pues, referencias ineludibles la Ley de Integración Social del Minusválido (LISMI) aprobada en 1982, el Plan Gerontológico de principios de los años 90, la II Asamblea Mundial de Envejecimiento de Naciones Unidas celebrada en España en 2002 y diferentes medidas legislativas que de una u otra forma han dado respuesta a situaciones de dependencia. Concretamente la Ley General de Sanidad (1986), la Ley de Pensiones No Contributivas (1990), la Ley General de la Seguridad Social (texto refundido de 1994, TRLGSS), Leyes Autonómicas de Servicios Sociales, la Ley de Cohesión y Calidad del Sistema Nacional de Salud (2003), así como el Plan Concertado para el Desarrollo de Prestaciones Básicas de Servicios Sociales de Comunidades Locales (1988).

En consonancia con los artículos 49 y 50 de la Constitución Española de 1978, desde los años 80 se han ido articulando los siguientes tipos de reformas, prestaciones y deducciones fiscales orientadas a dar cobertura a determinadas situaciones de envejecimiento y de discapacidad (Rodríguez Cabrero 2004; Rodríguez Rodríguez 2006): 
i) El punto de inflexión en la introducción del debate sobre una atención más integral, sociosanitaria y colectiva de la dependencia lo marcaron, a pesar de su insuficiente dotación presupuestaria y de la falta de compromiso político, tanto la Ley 13/1982, de Integración Social de los Minusválidos (LISMI) de 1982 como, sobre todo, la aprobación del Plan Gerontológico en 1992.

En cuanto a la primera, tuvo una orientación sesgada hacia las prestaciones económicas en detrimento de las políticas relativas a la integración sociolaboral o a la supresión de barreras arquitectónicas. No obstante, ya contemplaba pioneramente en su artículo 16 un subsidio de ayuda por tercera persona (suprimido en 1994) orientado a personas con 18 o más años sin actividad laboral, con un grado de discapacidad igual o superior al $75 \%$ y que necesitaran la asistencia de otra persona para realizar los actos más esenciales de la vida. En lo que respecta al Plan Gerontológico, por primera vez se abordaron de manera integral toda una serie de políticas en materia de envejecimiento más allá de sus efectos sobre las pensiones abriéndose camino la importancia de su atención sociosanitaria.

Sucede, empero que estas medidas, que tuvieron que ver lógicamente con la dependencia (aunque dicho término no se acuñó entonces), en su mayoría no se implementaron por la falta de financiación e impulso político y por la fragmentación de competencias entre las Administraciones públicas (Rodríguez Rodríguez 2006).

No obstante, de haberse llevado a cabo habrían supuesto un importante avance en la atención de situaciones de dependencia de personas mayores (Sancho y Rodríguez 2002). Por entonces ya contemplaban medidas como alcanzar ratios suficientes en los principales servicios de atención, un complemento de pensión para pensionistas en situación de dependencia funcional, o mapas sociosanitarios y unidades específicas para coordinar los servicios sociales y sanitarios.

ii) A nivel mesogubernamental destacan, hasta la elaboración de la LAPAD, tanto las leyes autonómicas de servicios sociales como el Plan Concertado para el Desarrollo de Prestaciones Básicas de Servicios Sociales de Comunidades Locales desde 1988.

Las leyes autonómicas de servicios sociales de primera y segunda generación (Guillén 2005) han ido configurando, junto a diversos programas sociosanitarios y planes de atención a personas mayores, un mapa autonómico de servicios sociales cada vez más extenso. Bien es cierto que, aunque "se proclaman como universales, es decir, accesibles a cualquier persona en situación de necesidad [...] se trata de un derecho puramente retórico, que no puede compararse de ninguna manera al derecho subjetivo que tienen los sistemas de protección ya consolidados" (Rodríguez Rodríguez 2006:50). En cuanto al Plan Concertado resulta significativo cómo en 1993 la Federación Española de Municipios y Provincias (FEMP) aprobó un catálogo en materia de servicios sociales. Este 
catálogo supuso la atribución de mayores competencias para las corporaciones locales en la extensión de servicios sociales, como la ayuda a domicilio y los cuidados de proximidad, así como mayores dotaciones de profesionales municipales.

iii) En el ámbito de la Seguridad Social, la Ley General de la Seguridad Social (Texto Refundido de 1994, TRLGSS) regula la incapacidad permanente como la gran invalidez, aplicada a los trabajadores afectados por una incapacidad permanente que necesiten la asistencia de otra persona para desarrollar actos básicos de la vida diaria. De ser así, tendrían derecho "a una pensión vitalicia incrementándose su cuantía con un complemento, destinado a que el inválido pueda remunerar a la persona que le atienda... (Art. 139.5). Igualmente, el artículo 181 (a) reconoce una asignación económica por cada hijo menor de 18 años 0 , cuando siendo mayor de dicha edad, esté afectado por una discapacidad, en un grado igual o superior al 65 por 100, a cargo del beneficiario.

En cuanto a las prestaciones de carácter no contributivo, se incorporaron a la Ley General de la Seguridad Social con la Ley de Prestaciones no Contributivas (1990). De este modo se extendieron los beneficios de la Seguridad Social a todas las personas sin recursos que, por razones de edad, accidente o enfermedad, no pudiesen trabajar, aunque no hubiesen cotizado lo suficiente para tener derecho a las pensiones y otras prestaciones de carácter contributivo. Asimismo, pasaron a contemplarse: (a) las prestaciones económicas por hijo a cargo mayor de 18 años y con minusvalía igual o superior al 65\% (Art. 181-184); (b) la "gran invalidez" (instituida por Ley 26/1990, de 20 de diciembre, de Prestaciones no Contributivas); y (c) la ayuda de tercera persona (Ley 13/1982, de Integración Social de los Minusválidos).

iv) Desde el ámbito sanitario, es preciso reseñar igualmente otras medidas como la Ley General de Sanidad (1986) que supuso la práctica universalización de la atención sanitaria a toda la población, y la Ley 16/2003 de Cohesión y Calidad del Sistema Nacional de Salud, que reguló por primera vez la llamada prestación socio-sanitaria. Esta prestación, sin remitirse exclusivamente a la dependencia, fue relevante para esta, ya que incluye prestaciones de cuidados de larga duración, atención sanitaria de la convalecencia, y rehabilitación en pacientes con déficit funcional recuperable. Según su artículo 14 tales prestaciones estarían destinadas a "aquellos enfermos, generalmente crónicos, que, por sus especiales características, puedan beneficiarse de la actuación simultánea y sinérgica de los servicios sanitarios y sociales para aumentar su autonomía, paliar sus limitaciones o sufrimientos y facilitar su reinserción social". No obstante, a pesar de este avance legislativo, su aplicación concreta aún no ha tenido lugar. 
v) Entre las ventajas fiscales, incluidas las de las Comunidades Autónomas en los respectivos tramos del IRPF, mencionar diversas medidas de conciliación de la vida familiar y laboral en la Ley 39/1999 por las que se puede reclamar una reducción de jornada o una excedencia con reserva de puesto de trabajo para el cuidado de niños o personas adultas en situación de dependencia. Ello se realiza sin remuneración pero manteniéndose el período de cotización efectiva, a efectos de las prestaciones de Seguridad Social por jubilación, incapacidad permanente, muerte o supervivencia y maternidad (Art. 180 del Texto Refundido de la Ley General de la Seguridad Social, TRLGSS) (Rodríguez Rodríguez 2006).

Las reformas sociales descritas han propiciado que la atención a la dependencia no sea una novedad en el ordenamiento jurídico. También han contribuido a situar a esta contingencia en la primera línea de la política social tras un largo proceso de debate iniciado a principios de la década de los años 90 . No obstante, además de estas reformas, también destacan una serie de condicionantes que -previos a la crisis económica iniciada en 2008-, han condicionado la reforma de la política social de atención a la dependencia en los últimos años.

b) Uno de estos condicionantes es el intenso cambio sociodemográfico acontecido en la sociedad española en los últimos años. Por un lado, es significativo el aumento de ciertas causas sobrevenidas de dependencia, fruto de un modo de vida en el que se asumen mayores riesgos (accidentes de tráfico 0 accidentes laborales, por ejemplo). De igual modo, el "coste del éxito" asociado a un creciente envejecimiento, en el que se han reducido más los factores de muerte que los factores de riesgo de dependencia, puso de manifiesto la fragilidad e insuficiencia del modelo vigente de atención a la dependencia. Pero, sin duda los cambios más sustanciales son aquellos que están influyendo en una reducción de la oferta tradicional de cuidados de que disponían las personas dependientes en décadas anteriores, en particular, el menor tamaño de las familias y la creciente participación de la mujer en el mercado laboral. Hay que decir, no obstante, que ello no implica un retroceso del modelo de atención familiar de cuidados sino una profunda reestructuración social interna.

c) Otro factor que hay que tener en cuenta es la transformación producida en la ideología sanitaria debida a la creciente relevancia atribuida a la prevención y a la integración en la comunidad de los colectivos con problemas de salud mental. Ello dio lugar a la destrucción de las redes de atención específicas para enfermos crónicos principalmente en los hospitales psiquiátricos, pero también en otra clase de enfermedades, trasladando así el "problema" y las soluciones de la dependencia a la sociedad y teniendo esta que hacerle frente de manera más integral y alternativa a la convencional. 
d) Por último, y no menos importante, es la estructura de organización y provisión de los cuidados la que ha condicionado la actual configuración del episodio de cambio analizado. Dicha estructura podría calificarse como de pluralista, familiar y multinivel, altamente intensiva en cuidados familiares, e insuficiente y desigual en oferta y cobertura de servicios públicos y en servicios privados de responsabilidad pública.

El carácter familiar del sistema de cuidados a personas dependientes se confirma por ser las familias las primeras proveedoras de cuidados tal y como se pone de manifiesto en la Encuesta de condiciones de vida de los mayores 2006 (Imserso-CIS 2006). Según esta encuesta, mientras los cuidadores principales familiares (cónyuge, hijas e hijos) suelen representar, por término medio, el $71 \%$ de los cuidados principales, los servicios sociales públicos o las empresas de servicios sociales privadas tan solo representan el 2,5 y el $0,2 \%$ respectivamente.

Del mismo modo, se constata que desde las AAPP existe un amplio mapa de prestaciones y ventajas fiscales para atender esta contingencia. No obstante, pese a haber aumentado significativamente la oferta y cobertura de servicios sociales en los últimos años, dichas prestaciones son insuficientes y están desequilibradas territorialmente en tasas de cobertura, en requisitos de acceso y en aportaciones del usuario (Informe del Defensor del Pueblo 2000). Véanse por ejemplo, los Centros de día en 2008 donde contrastan las tasas de cobertura de Madrid $(1,28)$ o Cataluña $(1,07)$, con las de Asturias $(0,56)$, Galicia $(0,57)$, Navarra $(0,57)$ o Castilla y León $(0,58)$, (Imserso 2009).

Dichas prestaciones se establecen en función de la cuantía de la pensión (como sucede con las pensiones contributivas de gran invalidez) y no del grado de dependencia, las cuales pueden resultar en cierta medida discriminatorias y poco equitativas por cuanto dejan fuera a una buena parte de las personas con dependencias (menores de 18 años y mayores de 65). En el caso de las desgravaciones fiscales, estas excluyen a aquellos contribuyentes que no hacen la declaración de la renta 0 a los cuidadores que no conviven con el dependiente.

Este mapa de prestaciones, ha puesto de manifiesto con sus limitaciones la necesidad de llevar a cabo una reforma como la LAPAD, pero también ha permitido sentar las bases para que dicha reforma pueda implementarse sin partir de la nada.

El análisis realizado hasta ahora avala que la política social de atención a la dependencia en España responde a la acumulación y confluencia de los factores de índole socioeconómica, organizativa e institucional descritos, los cuales han ido propiciando una reforma gradual de la política social de atención a la dependencia en España hasta alcanzar su máxima expresión normativa en la LAPAD.

De este modo, parece quedar demostrada la primera hipótesis planteada de que la LAPAD no supone un episodio de cambio radical o abrupto, sino una reforma gradual dependiente de una senda (path dependency) acumulativa de factores socioeconómicos e institucionales acaecidos desde los años 80 , sin los cuales no habría sido posible el actual modelo de atención a la dependencia. 
LA POSICIÓN DE LOS ACTORES SOCIALES EN EL DESARROLLO DE LA LAPAD: LOS EJES BÁSICOS DEL DEBATE

\section{Antecedentes del papel de los actores sociales en el debate de la dependencia}

El papel de los actores sociales no es un fenómeno nuevo en el ámbito de la dependencia ni comienza con el proceso de debate de la LAPAD. La creciente "densidad de conciencia" de los distintos actores sociales sobre el fenómeno de la dependencia tiene un carácter acumulativo que parte de los años 1980, de un "movimiento ilustrado" minoritario. Este "movimiento" estuvo encabezado por expertos y académicos y por las clases profesionales procedentes del ámbito de la Seguridad Social y de la geriatría y la gerontología, con amplios conocimientos sobre las experiencias de otros países en dicho campo.

Sus principales aportaciones fueron, por un lado, contribuir a crear una "conciencia por goteo" sobre dicho fenómeno trasladando sus inquietudes a las administraciones públicas, las cuales reaccionaron con cierto retraso a las demandas sociales latentes que estaban emergiendo. Por otro lado, dicho movimiento ilustrado, en especial las clases profesionales procedentes del ámbito de la geriatría y la gerontología, fueron sintetizando progresivamente una demanda social procedente de un creciente número de familias sobrepasadas por el aumento de la esperanza de vida con elevados años de discapacidad, y plenamente concienciadas del problema (Marbán 2009).

Posteriormente, a inicios de 2000 , las demandas sociales ${ }^{2}$ fueron canalizadas también, y en mayor medida, por los sindicatos y por las organizaciones sociales de la discapacidad y de personas mayores, a través de consejos estatales y plataformas de entidades (Plataforma de ONG de acción social, Consejo Estatal de personas Mayores, Consejo de ONG de Acción Social, Comité Español de Representantes de Personas con Discapacidad - CERMI). Ello se produjo con un cierto retraso respecto a los planteamientos articulados por expertos y profesionales debido a que, según algunos de los entrevistados, hasta los inicios del siglo XXI las prioridades de los sindicatos y de dichas organizaciones sociales no fueron la atención a la dependencia sino a otros tipos de reivindicaciones: en el caso de los sindicatos, las pensiones o la protección al desempleo; y en el caso de los movimientos sociales, la ampliación de programas de turismo y de termalismo, la subida de las pensiones y la integración laboral y educativa.

${ }^{2}$ El 76,1\% de los ciudadanos consideraban una prioridad inmediata la atención a las personas dependientes para aliviar la sobrecarga que sus cuidados suponen para las familias, (Centro de Investigaciones Sociológicas 2004). 


\section{Los ejes básicos del debate y sus implicaciones para los actores sociales}

A partir del encuadramiento teórico apuntado en la introducción, en el siguiente cuadro se han articulado los ejes básicos del debate en torno a la tríada ideas-intereses-instituciones:

En el plano institucional, destacar cómo buena parte del proceso discurrió a través de mecanismos institucionalizados, como el acuerdo social de 2005; el sometimiento del anteproyecto a dictámenes e informes independientes y, por último, su aprobación parlamentaria.

Para el "acuerdo social sobre la acción protectora de la atención a las situaciones de dependencia"s firmado entre patronal, sindicatos y gobierno en diciembre de 2005 se tomó como base de debate la elaboración del Libro Blanco de la Dependencia (publicado a principios de enero de 2005). En dicho acuerdo se acordaron aspectos básicos de lo que sería la futura LAPAD, como su desvinculación de la Seguridad Social, la forma de financiar esta política o la pretensión de priorizar los servicios más que las prestaciones económicas

En lo que respecta a los dictámenes e informes independientes al anteproyecto de ley destacan los del CES (Consejo Económico y Social 2006) y el Consejo de Estado (2006); las propuestas por parte de sindicatos, organizaciones sociales del ámbito de la discapacidad y de personas mayores, y diversos informes independientes (Sociedad Española de Geriatría y Gerontología 2006; Seminario de Intervención y Políticas Sociales 2006). Su impacto se notó en la introducción de varios cambios que se reflejarían después en el proyecto de ley, como la inclusión de pleno derecho de las personas con discapacidad intelectual y enfermedad mental, el fomento del empleo de las personas con discapacidad (Disposición adicional decimocuarta), la consideración del impacto de género en la LAPAD (Disposición final 1. ${ }^{\text {a }} 4$ ) o la cobertura a los menores de tres años con graves discapacidades (Disposición adicional decimotercera).

Por último, en cuanto a la aprobación parlamentaria de la LAPAD, rechazaron la reforma los partidos políticos CIU, PNV y EA (con la amenaza de inconstitucionalidad hasta el último momento) y se abstuvo Nafarroa Bai, argumentando todos ellos financiación insuficiente y que la ley invadía competencias exclusivas de las CCAA en materia de servicios sociales.

En el plano de las ideas-intereses se destacarían fundamentalmente dos situaciones:

I. Por un lado, aquellos aspectos sobre los que predominó el consenso debido a la confluencia de ideas articuladas sobre intereses conciliables, a veces competitivos (por ejemplo, provisión pública y privada lucrativa y no lucrativa). Concretamente, el grado de acuerdo sobre lo que debería ser la LAPAD fue sustancial en ideas básicas como su uni-

\footnotetext{
${ }^{3}$ Los detalles del acuerdo pueden consultarse en: http://www.imsersomayores.csic.es/documentacion/ documentos/registro.jsp?id=1375
} 


\section{Cuadro 1. La tríada instituciones-ideas-intereses en el debate entre actores sociales implicados en la LAPAD.}

\begin{tabular}{|c|c|c|c|c|c|}
\hline 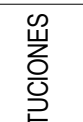 & IDEAS & & INTERESES & & \\
\hline & & Sindicatos & OTS & CCAA & Patronal \\
\hline$\frac{\mathscr{e}}{\frac{\mathbb{\pi}}{\mathrm{co}}}$ & $\begin{array}{l}\text { Reconocimiento de } \\
\text { los derechos de los } \\
\text { cuidadores en la } \\
\text { Seguridad Social }\end{array}$ & $\begin{array}{c}\text { Reconocimiento } \\
\text { papel de } \\
\text { cuidadores } \\
\text { Reducir su } \\
\text { precariedad }\end{array}$ & $\begin{array}{l}\text { Reconocimiento papel de } \\
\text { cuidadores } \\
\text { Reducir su precariedad }\end{array}$ & $\begin{array}{c}\text { Reconocimiento } \\
\text { papel de } \\
\text { cuidadores } \\
\text { Reducir su } \\
\text { precariedad }\end{array}$ & - \\
\hline 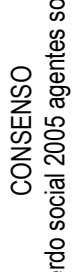 & $\begin{array}{l}\text { Universalidad } \\
\text { Preferencia por } \\
\text { un modelo de } \\
\text { prestaciones en } \\
\text { servicios antes } \\
\text { que por un modelo } \\
\text { de prestaciones } \\
\text { monetarias } \\
\end{array}$ & $\begin{array}{l}\text { Consolidación } \\
\text { provisión servicios } \\
\text { públicos }\end{array}$ & $\begin{array}{c}\text { Consolidación oferta no } \\
\text { lucrativa }\end{array}$ & $\begin{array}{l}\text { Consolidación } \\
\text { red de servicios } \\
\text { sociales }\end{array}$ & $\begin{array}{c}\text { Consolidación } \\
\text { oferta privada } \\
\text { lucrativa de } \\
\text { servicios de } \\
\text { atención }\end{array}$ \\
\hline 岂 & $\begin{array}{c}\text { Potenciar la } \\
\text { prevención, la } \\
\text { formación, la calidad } \\
\text { en la prestación de } \\
\text { servicio }\end{array}$ & $\begin{array}{l}\text { Implicación en la } \\
\text { oferta de formación } \\
\text { Mejora de la } \\
\text { calidad del servicio }\end{array}$ & $\begin{array}{l}\text { Implicación en la oferta } \\
\text { de formación } \\
\text { Mejora de la calidad del } \\
\text { servicio }\end{array}$ & $\begin{array}{l}\text { Mejora de la } \\
\text { calidad del } \\
\text { servicio }\end{array}$ & $\begin{array}{c}\text { Mejora de la } \\
\text { calidad del } \\
\text { servicio }\end{array}$ \\
\hline 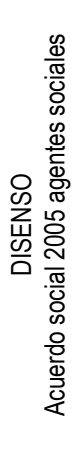 & $\begin{array}{c}\text { Marco } \\
\text { protector-financiación- } \\
\text { ámbito competencial } \\
\text { Seguridad Social } \\
\text { Impuestos }\end{array}$ & $\begin{array}{l}\text { Recentralización } \\
\text { de servicios } \\
\text { sociales; mayor } \\
\text { garantía de } \\
\text { igualdad de acceso } \\
\text { y sosteniblidad en } \\
\text { todo el territorio y } \\
\text { de extensión de los } \\
\text { servicios públicos }\end{array}$ & $\begin{array}{l}\text { Mayor garantía de } \\
\text { igualdad de acceso y } \\
\text { sosteniblidad en todo el } \\
\text { territorio, financiación más } \\
\text { estable garantizada para } \\
\text { extensión de los servicios } \\
\text { privados no lucrativos de } \\
\text { responsabilidad pública }\end{array}$ & $\begin{array}{l}\text { Conservar } \\
\text { competencias, } \\
\text { Identificación } \\
\text { directa del } \\
\text { derecho } \\
\text { subjetivo }\end{array}$ & $\begin{array}{l}\text { Contención } \\
\text { de nuevas } \\
\text { cotizaciones }\end{array}$ \\
\hline 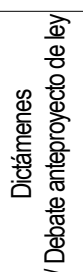 & $\begin{array}{l}\text { Ampliación a } \\
\text { colectivos }\end{array}$ & $\begin{array}{c}\text { Contener costes } \\
\text { SAAD }\end{array}$ & $\begin{array}{c}\text { Ampliación de cobertura } \\
\text { Fomento de la auto- } \\
\text { nomía }\end{array}$ & $\begin{array}{l}\text { Contener } \\
\text { costes SAAD }\end{array}$ & $\begin{array}{l}\text { Contener } \\
\text { costes } \\
\text { SAAD }\end{array}$ \\
\hline
\end{tabular}


versalidad, el reconocimiento de los derechos de los cuidadores en la Seguridad Social, la preferencia por un modelo de prestaciones por servicios antes que por un modelo de prestaciones monetarias, la importancia concedida a la prevención, la formación, la calidad en la prestación de servicios o la necesidad de establecer un sistema de información transparente y fluido entre los actores implicados.

En aspectos como el reconocimiento de los derechos de los cuidadores en la Seguridad Social y la importancia concedida a la prevención y la calidad en la prestación de servicio, los intereses de los actores sociales no entraban en conflicto entre sí por la coincidencia de todos ellos en la necesidad de mejorar la calidad del servicio, el reconocimiento del papel de los cuidadores 0 en reducir su precariedad.

En cuanto a otros aspectos más instrumentales, como las posibilidades abiertas para los actores sociales en la provisión de servicios, sus intereses, aunque no fueran coincidentes, eran compatibles; en la medida en que, con la LAPAD no se descartaba una provisión pluralista de servicios públicos, privados lucrativos y no lucrativos.

II. Por otro lado, cabe destacar aspectos como el triple eje marco protector-financiaciónámbito competencial, y los colectivos incluidos en la LAPAD, sobre los que se constata la divergencia en ideas articuladas sobre intereses contrapuestos entre los actores sociales.

1. En cuanto al marco protector-financiación-ámbito competencial, el debate sobre la inclusión de la ley en el marco de la Seguridad Social, siguiendo el modelo alemán, 0 bien, fuera de ella, optando por ser financiada vía impuestos como en el sistema nórdico, fue intenso entre los diferentes actores sociales.

Las razones por las que se decidió finalmente excluir la ley del ámbito de la Seguridad Social y financiar su implementación con impuestos no fueron del todo justificadas por los responsables políticos. No obstante, del análisis de los discursos de los actores sociales implicados en esta investigación se desprende que, por un lado, mientras los sindicatos, una buena parte de los expertos consultados y las organizaciones del Tercer Sector eran proclives a la idea de incluir los derechos y prestaciones en el ámbito de la Seguridad Social, por otro lado, la patronal, gran parte de las CCAA y los partidos políticos de corte más autonomista se oponían.

Como hemos comentado una parte sustancial de la decisión se gestó en el acuerdo social entre los sindicatos, la patronal y el gobierno como consecuencia de la presión de la patronal, contraria a un incremento de las cotizaciones. La principal razón por la que los sindicatos cedieron en este punto fue que, al querer priorizarse en la atención a la dependencia las prestaciones en servicios sobre las prestaciones económicas, su integración en la Seguridad Social podría implicar un replanteamiento del Pacto de Toledo, cuya dinámica era la de sacar del sistema contributivo todo lo que no fuera protección económica "pura y dura".

Conviene apuntar que la validez de este argumento está siendo puesta en entredicho tanto por el propio desarrollo de la ley como por los propios expertos entrevistados. 
Aunque todavía se está en sus inicios, la realidad de la implementación de la Ley de Dependencia en sus más de tres años ha demostrado que la falta de servicios sociales apropiados la está convirtiendo en una ley de subsidios y prestaciones económicas que, de momento, bien podría haber encajado en la Seguridad Social. Del mismo modo, se entiende que el argumento de la patronal de que la inclusión de la Ley en el marco de la Seguridad Social aumentaría las cotizaciones tampoco estaría justificado, por cuanto podría haberse incluido dentro de la rama no contributiva y, por tanto, tener una financiación fiscal separada.

De esto se puede inducir que su exclusión del marco de la Seguridad Social no se debió tanto al frágil argumento de la patronal de que con ello aumentarían las cotizaciones, sino fundamentalmente a las implicaciones competenciales que ello conllevaría para las CCAA y para los partidos políticos más autonomistas (los cuales tuvieron una mayor presencia parlamentaria durante la legislatura en que se aprobó la Ley). Según estos actores sociales, incluir la Ley de Dependencia en el sistema de la Seguridad Social conferiría al Gobierno central una capacidad imperativa que invadía las competencias de las CCAA en servicios sociales y que les restaba legitimidad para intervenir en la práctica.

Puede argüirse por lo tanto, que la exclusión de la Ley de Dependencia del marco de la Seguridad Social estuvo motivada no solo por razones de financiación, sino especialmente por razones de tipo competencial entre los niveles central y autonómico del Estado español. De las entrevistas realizadas, no se puede asegurar si su exclusión se produjo con la connivencia del gobierno del PSOE en la medida en la que pudieran prevalecer en su decisión los criterios de financiación (compartida con las CCAA) por encima de los competenciales. En cualquier caso, y de acuerdo con la visión de algunos expertos, el haber situado la Ley de Dependencia fuera de la Seguridad Social ha supuesto para la Administración Central el tener que pactar con todas y cada una de las Comunidades Autónomas. Con ello se habría perdido la oportunidad de poner una "banderita" en los servicios sociales como competencia propia, lo que la Constitución de 1978 permite plenamente al Gobierno central.

2. En cuanto a los colectivos incluidos en la Ley también fueron objeto de un intenso debate especialmente por parte de las organizaciones de la discapacidad. Éstas abogaban por la inclusión en la ley de colectivos inicialmente no previstos en el anteproyecto de ley, como las personas con discapacidad intelectual y enfermedad mental, y su reflejo en el baremo de medición de la dependencia. También planteaban la incorporación en la ley de las necesidades de apoyo y promoción de la autonomía personal.

La patronal, sindicatos o CCAA no se opusieron frontalmente pero se mostraron escépticos por cuanto de ampliación de la LAPAD a colectivos inicialmente no previstos podría encarecer la implementación de la LAPAD.

Por el contrario, las organizaciones no lucrativas de la discapacidad vieron en esta ley una posibilidad de cumplir unas expectativas que no habían sido cubiertas con otras leyes o planes, como los planes nacionales de empleo, en temas de discapacidad o la 
ley de igualdad de oportunidades. Ello propició que este sector se "agarrase" al tren de la universalización subjetiva de esta contingencia poniendo en funcionamiento una capacidad de movilización y de negociación que había permanecido "aletargada" hasta finales de los años 90.

Las propuestas desde este sector de asociaciones de personas con discapacidad y personas mayores fueron frecuentes, especialmente desde el CERMI, la Plataforma de ONG de Acción Social, la Confederación Española de Organizaciones en favor de las Personas con Discapacidad Intelectual (FEAPS), la Confederación Española de Organizaciones de Mayores (CEOMA), la Unión Democrática de Pensionistas (UDP) 0 el Foro de Vida Independiente. Finalmente, consiguieron la inclusión en la ley de los mencionados colectivos fundamentalmente por la capacidad instalada de este sector en la provisión de servicios y por la legitimación social de su propuesta.

De lo analizado hasta ahora podemos constatar la importancia de los actores colectivos en la configuración de la reforma especialmente en aspectos como el marco protector, la financiación y el nivel competencial, en donde distintas combinaciones de actores sociales comparten ideas y buscan la consecución de una serie de objetivos comunes en la configuración de la actual LAPAD.

Hemos analizado cómo, por un lado, las organizaciones del TS y los sindicatos eran proclives a la idea de incluir los derechos y prestaciones en el ámbito de la Seguridad Social y a una participación más activa de la Administración Central en los servicios sociales. Con ello se respondía con mayores garantías a sus principales intereses, en particular, la igualdad de acceso en todo el territorio, la extensión de los servicios públicos y la extensión de los servicios públicos y privados no lucrativos de responsabilidad pública. No obstante, a pesar de los puntos de encuentro, no cabría hablar de una verdadera coalición entre organizaciones del TS y sindicatos. Del análisis de las entrevistas, se observa cómo en el proceso pesaron más sus ya "tradicionales" diferencias, ancladas en la lucha subyacente por ocupar espacios de acción social comunes, que la búsqueda de apoyos mutuos en la consecución de un determinado modelo de atención a la dependencia.

Por otro lado, la patronal, gran parte de las CCAA y los partidos políticos de corte más autonomista coincidian en la oposición a la idea de incluir la LAPAD en el marco de la Seguridad Social y abogaban por una financiación vía impuestos. No obstante, aunque sus intereses fueran compatibles, no fueron tan coincidentes como para propiciar una coalición formal entre ambos: para las CCAA y para los partidos políticos de corte más autonomista, el incluir la LAPAD en el sistema de la Seguridad Social era una cuestión competencial pues conferiría al Gobierno central una capacidad imperativa que invadía las competencias de las CCAA en servicios sociales y que les restaba legitimidad para intervenir en la práctica. Para la patronal, la inclusión en la Seguridad Social fue una cuestión de carácter más económico ya que, de haberse conseguido, habría supuesto un aumento en sus costes laborales (vía cotizaciones sociales) lastrando así su crecimiento organizativo.

De todo ello, se puede inducir que, como apuntábamos en la segunda hipótesis, la LAPAD es el resultado de la importancia central de intensos procesos de negociación 
entre los distintos actores sociales. No obstante, no se puede afirmar con seguridad que para ello hayan establecido coaliciones basadas en estructuras organizativas y estrechas redes de contacto y cooperación mutua o alianzas diseñadas por acuerdo, sino más bien "coaliciones sui géneris" basadas en la confluencia inercial de intereses conciliables en mayor o menor grado entre distintas combinaciones de actores ${ }^{4}$.

\section{Tres años de LAPAd. Un análisis tentativo de los Resultados, Retos e INCERTIDUMBRES}

Ciertamente, puede resultar prematuro valorar los resultados de la Ley de Dependencia algo más de tres años después de su puesta en marcha en 2007 (en la práctica, tras el verano de 2007). No obstante, desde la puesta en marcha de la LAPAD en 2007 son muy destacables los esfuerzos realizados en los aspectos financiero, reglamentario, y prestacional (Asociación Estatal de Directores y Gerentes en Servicios Sociales 2009). Aunque el sistema será evaluado formalmente en 2010, informes de evaluación cómo el del Grupo de expertos (2009) para la evaluación del desarrollo y efectiva aplicación de la Ley 39/2006, en 2009, ya apuntan como el sistema está mejorando a medida que avanza su implementación. Hasta el 1 de abril de 2011 el número de dictámenes de dependencia alcanzaba a 1.443.327 personas y los beneficiarios con derecho a prestación a 1.071 .603 , siendo el total de personas beneficiarias con prestaciones de 721.000 , lo que supone ciertamente que el SAAD está adquiriendo paulatinamente su velocidad de crucero.

No obstante, en este breve recorrido tanto las personas entrevistadas como las fuentes secundarias y la práctica cotidiana de su implementación nos han permitido vislumbrar algunos problemas, retos e incertidumbres que pesan sobre la ley y que están contribuyendo al debate sobre su futura reforma.

1. En lo referente al ámbito competencial y al gobierno del sistema de atención a la dependencia, se sustenta en un tipo de gobernanza de carácter mixto y cooperativo entre las CCAA y la Administración General del Estado (AGE) a tres niveles: el Consejo Territorial del Sistema para la Autonomía y Atención a la Dependencia (SAAD), los convenios de colaboración bilaterales y los planes conjuntos.

Ante la inexistencia de una ley marco de servicios sociales, este tipo de gobernanza requiere una ágil y leal colaboración entre las distintas administraciones públicas impli-

\footnotetext{
${ }^{4}$ Esta ausencia de coaliciones entre los actores sociales coincide además con la respuesta dada por la mayoría de actores sociales entrevistados cuando se les planteó la pregunta: "¿se formaron "coaliciones" de algún tipo a lo largo del proceso?". La mayoría manifestó no percibir ningún tipo de coalición ni estructura formal de cooperación con otros actores sociales.
} 
cadas en el proceso, con el fin de garantizar los principios de acceso y calidad del sistema a toda la población y para reducir unos costes de transacción que por la vía del convenio anual entre las CCAA y la AGE se prevén excesivamente altos.

Aunque todavía resulta prematura la evaluación del sistema, la capacidad regulatoria de la ley no garantiza la homogeneidad del sistema en el acceso y calidad de las prestaciones en todo el territorio español ya que el SAAD se integra dentro del sistema de servicios sociales de las CCAA cuya diversidad es manifiesta y está basada en su competencia exclusiva en la materia. El Estado puede contribuir a equilibrar el sistema garantizando una prestación mínima igual per capita en todo el territorio del Estado, así como contribuir a cierta redistribución territorial según la prestación acordada, que se basa en criterios de población, personas atendidas, dispersión territorial e insularidad, con el fin de garantizar cierta equidad en un sistema diverso por definición. Pero al no sustentarse en una ley básica no puede alterar el equilibrio competencial.

En este sentido cabe afirmar que existen tantos sistemas de atención a la dependencia como CCAA. Del mismo modo, la implementación de la ley en sus tres primeros años parece estar produciéndose a distintas velocidades entre las CCAA tal y como han puesto de manifiesto distintos dictámenes de la Asociación Estatal de Directores y Gerentes en Servicios Sociales (AEDGSS 2009).

Estas diferencias en los ritmos de implementación podrían venir explicadas por distintos factores como, entre otros, limitaciones en el sistema de información entre la AGE y las CCAA sobre los dictámenes y prestaciones reconocidas, los cambios de gobierno autonómicos asociados a procesos electorales, retrasos en la reglamentación autonómica específica para aplicar la ley, diferentes niveles de infraestructuras asistenciales asociados al modelo de servicios sociales de cada CCAA o la falta de protocolos de participación de los ayuntamientos en la gestión de servicios de atención. De igual modo, se ha puesto de manifiesto en otros trabajos como el factor políticoideológico también parece influir en la velocidad de implementación de la ley aunque ciertamente no resulte determinante (Marbán 2009).

La Ley de Dependencia estaría sometida así a las coyunturas sociopolíticas general y de las CCAA cuya tendencia centrífuga del sistema de atención a la dependencia sólo puede ser frenada con un papel activo de los Consejos Consultivos ${ }^{5}$, a juicio de un experto académico entrevistado.

Ello explica en cierto modo que no exista homogeneidad territorial en el SAAD en lo relativo al acceso, acreditación de centros y copago. La propia aplicación del baremo de valoración, siendo un instrumento único en todo el Estado, queda mediatizada por la composición de los equipos de valoración, cuya diversidad es manifiesta. Incluso la definición del PIA (programa individual de atención), instrumento de materialización de las prestaciones reconocidas, se desarrolla en cada CCAA de acuerdo con su tradición de servicios sociales y estilo específico de intervención profesional. De ahí la afirmación

\footnotetext{
${ }^{5}$ Los órganos consultivos se mencionan en los artículos 40 y 41 de la Ley 39/2006.
} 
antes señalada de que el sistema español de dependencia es, en realidad, un conjunto de 17 sistemas relativamente coordinado.

2. Es recurrente en los discursos de los actores sociales entrevistados la mención a la incertidumbre financiera que pesa sobre la ley como consecuencia de varios factores y cuyos efectos pueden tener consecuencias en una baja intensidad protectora.

La suficiencia financiera del sistema planea como un déficit importante de la LAPAD porque los costes reales de atender a una persona gran dependiente son mayores de los previstos. También porque las estimaciones de población dependiente se están viendo ampliamente sobrepasadas por la realidad como consecuencia de: 1) la ampliación de la ley a colectivos inicialmente no contemplados ni en el proyecto de ley ni en el Libro Blanco, 2) por la aplicación discrecional del baremo de valoración de las personas dependientes o 3) simplemente por una errónea estimación de la población beneficiaria. En este sentido, se debe tener en cuenta que, a fecha julio de 2009, las personas dictaminadas como dependientes de grado III (en concreto, 402.805) ya prácticamente habían duplicado las proyecciones realizadas en el Libro Blanco para el año 2010, estimadas en 223.457, y ya superan ampliamente las previsiones de población dependiente de grado III en 2007 de 206.000 personas.

Esto puede tener una repercusión importante en la sostenibilidad del sistema, máxime si se tiene en cuenta que, según está evolucionando el sistema, las primeras estimaciones de coste (9.4 millones de euros para el año 2015) serán ampliamente sobrepasadas a medio plazo (14,4 millones en dicho año) (Montserrat Codorniu 2007).

3. Aun cuando la ley contempla todo lo contrario, una consecuencia no prevista del sistema de dependencia es la sostenida prevalencia de las prestaciones económicas sobre las prestaciones en servicios. En concreto, el peso de las prestaciones económicas para cuidados en el entorno familiar sobre el total de prestaciones reconocidas entre diciembre de 2008 y noviembre de 2010 ha aumentado del $46,5 \%$ al $49 \%$ mientras que el peso de las prestaciones en servicios concretos como ayuda a domicilio han descendido del $14 \%$ al $11 \%$ y en centros de día/noche apenas han aumentado por encima del $5 \%$ (gráfico l).

En el caso de España, la prevalencia de las prestaciones económicas podría explicarse por: (a) razones económicas, al ser más "baratas" que las prestaciones en servicios; (b) la facilidad de la gestión para las propias administraciones; (c) por las citas electorales acontecidas durante su desarrollo y durante las cuales se iban asignando prestaciones económicas, dada su mayor visibilidad y ágil concesión, y (d) porque pueden contar con cierto consenso social por parte del núcleo principal de cuidadores, mujeres de entre 55 y 70 años con poca cualificación profesional que, o no han estado nunca en el mercado laboral, o han estado poco tiempo.

Esta prevalencia de las prestaciones económicas por el momento puede estar produciendo algunos efectos negativos sobre la calidad de los cuidados, ya que en los grados de dependencia muy severos la prestación más adecuada probablemente no sea 
Gráfico I.

Evolución del peso de las prestaciones económicas y en servicios sobre el total de prestaciones reconocidas e identificadas

en el PIA. Diciembre 2008-noviembre 2010 (\%)

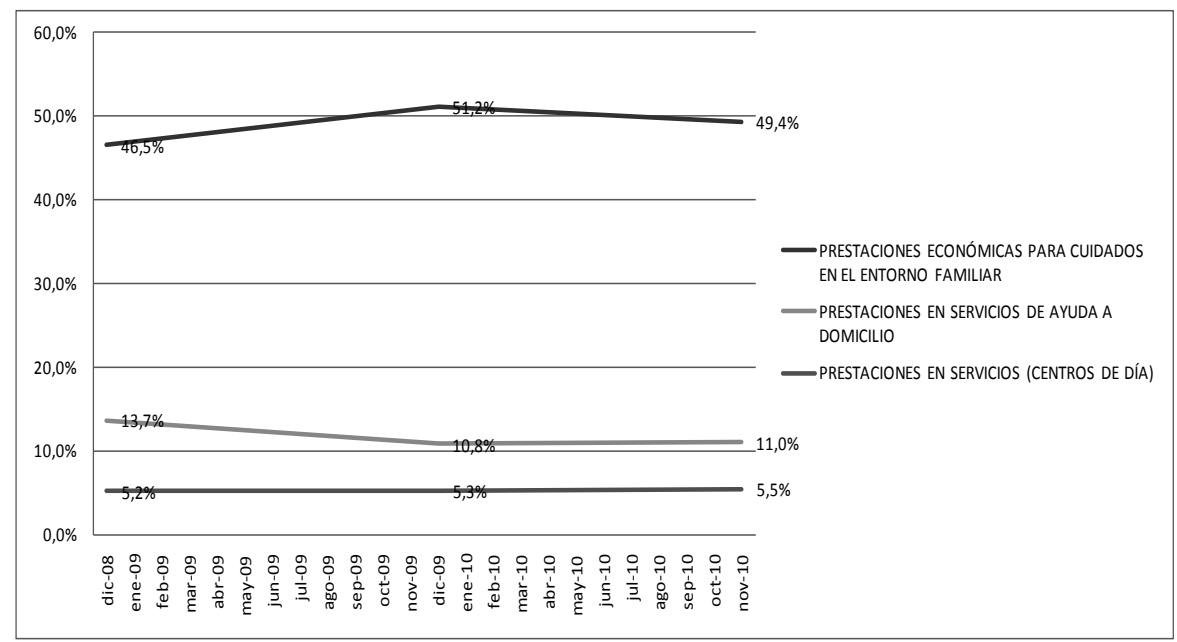

Fuente: Elaboración propia a partir de SAAD-IMSERSO.

una prestación económica para cuidados en el entorno familiar sino atención residencial. También afecta negativamente a las expectativas de quienes pensaban que con la ley se reforzaría la red de servicios existente: a los sindicatos, por cuanto con ello no se está generando empleo en la provisión de dichos servicios; a los empresarios, en la medida en la que la prestación no se vincula a un servicio que ellos podrían ofertar.

Este "voto" mayoritario en favor de las prestaciones económicas conduce a una necesaria reflexión sobre la persistencia del modelo de cuidados español, del tipo mediterráneo, que lejos de desaparecer se está transformando más lentamente de lo previsto. Los bajos niveles de la oferta de servicios sociales son un argumento importante pero no el único y el definitivo. Las tradiciones culturales, el mayor apoyo a las prestaciones económicas frente a los servicios en la cultura de protección social española y, actualmente, el efecto de la crisis económica, han hecho de la excepcionalidad de las prestaciones económicas prevista en la LAPAD la norma social prestacional.

4. La coordinación sociosanitaria ha supuesto uno de los déficit de esta Ley en la medida en la que no se ha abordado plenamente. Según la visión de expertos y de representantes gubernamentales, la Ley ha quedado reducida a un servicio social bien sea por acción u omisión del equipo de gobierno central, por una falta de liderazgo compartido entre los ministerios implicados, o bien, por los distintos niveles de desarrollo, organización y prácticas profesionales que existen entre lo sanitario y lo social. 
En este sentido, la Ley de Dependencia podría constituir una oportunidad desaprovechada para mejorar la coordinación sociosanitaria. Esto convierte a la Ley de Dependencia, en expresión de un entrevistado, en "un coloso de Rodas con una sola pierna...", contradiciendo así a la Ley de Cohesión y Calidad del sistema sanitario de 2003, que contemplaba las prestaciones sociosanitarias y que no se han desarrollado en el nuevo catálogo de prestaciones sanitarias de 2006.

5. De igual manera se hace necesaria una mejora en los sistemas de información de la atención a la dependencia. No solo en lo referente a la red de comunicaciones y servicios telemáticos del sistema, sino también a la información suministrada por las CCAA en lo relativo a las prestaciones reconocidas. Con los datos proporcionados por las CCAA a 1 de abril de 2011, el 16\% de las prestaciones reconocidas están pendientes de identificar en el Plan Individualizado de Atención, es decir, que no se especifica el tipo de prestación recibida. Esto nos puede hacer suponer que no necesariamente todas las personas que tienen reconocida una prestación la están percibiendo todavía. Ello puede deberse, según los representantes de las CCAA, a que hay personas que todavía no tienen cumplimentado el PIA (Plan Individualizado de Atención), que no han elegido la ayuda deseada o no se ha determinado la prestación que recibirán, o bien, que simplemente han fallecido pero ya habían generado el derecho a la prestación.

En cualquier caso, supone un déficit del sistema que se pretende superar con la aprobación del Real Decreto 99/2009, de 6 de febrero. Con el mismo, se endurecen las condiciones para librar las transferencias a las CCAA del nivel mínimo de protección garantizado para cada beneficiario por la Administración General del Estado. Estas quedarían condicionadas a que la valoración se realice únicamente aplicando el baremo acordado por el Consejo Territorial, a que se indique expresamente el grado, nivel y los PIA aprobados de los beneficiarios, y a que no pase más de un año sin recibir la prestación desde la fecha de resolución.

\section{BREVES CONCLUSIONES Y PROPUESTAS TENTATIVAS}

La aprobación de la LAPAD supone un hito en la expansión del Estado de Bienestar en España por cuanto desarrolla un derecho universal subjetivo para todos los individuos que presenten un grado de dependencia (moderada, severa o gran dependencia) determinada según la frecuencia y la intensidad de la ayuda requerida.

A partir del contraste de las hipótesis analizadas en el texto podemos concluir que la LAPAD resultante y su implementación, es el resultado tanto de una senda acumulativa (path dependency) de factores socioeconómicos e institucionales acaecidos en la política social de atención a la dependencia desde los años 80 , como de intensos procesos de negociación entre los distintos actores sociales implicados. 
Ello implica afirmar, en primer lugar, que LAPAD no supone un episodio de cambio o una reforma radical sido una reforma gradual dependiente de la acumulación de los siguientes factores socioeconómicos e institucionales acaecidos desde los años 80 .

1) El contexto europeo, las reformas políticas y las inercias institucionales (Policy legacy and policy reforms) acaecidas en el ámbito de la dependencia desde los años ochenta (Plan Gerontológico, LISMI, Ley General de la Seguridad Social, etc.)

2) La estructura de organización y provisión de los cuidados en las esferas pública y privada (social caring setting) altamente intensiva en cuidados familiares e insuficiente y desigual en oferta y cobertura de servicios públicos y en servicios privados de responsabilidad pública.

3) Cambios sociodemográficos (envejecimiento, nuevas dinámicas familiares, incorporación de la mujer al mercado laboral).

En segundo lugar, se considera que este episodio de cambio es el resultado de la importancia central de los actores sociales cuyas ideas e intereses se han ido conjugando de modo inercial durante el diseño de la ley más que mediante estructuras organizativas y estrechas redes de contacto y cooperación mutua o alianzas diseñadas por acuerdo.

La LAPAD ha sido fruto de procesos de negociación intensos entre los distintos actores sociales implicados donde no todos los actores han salido igualmente reforzados. En términos generales, ha supuesto un reforzamiento de la posición de las organizaciones de la discapacidad y las CCAA, no tanto así del Estado central o los sindicatos.

En cuanto a las CCAA, han conseguido la preservación de sus competencias en servicios sociales frente al Estado central en la medida en la que la LAPAD queda fuera del régimen económico de la Seguridad Social y permite a las CCAA establecer un nivel adicional de protección más allá del nivel acordado con la AGE vía convenio. № obstante, tampoco cabe menospreciar el impacto que tendrá la LAPAD en los servicios sociales de las CCAA si se tiene en cuenta que tendrán que adaptar y revisar sus leyes de servicios sociales y que algunas de las decisiones en materia de dependencia tendrán que ser acordadas en el Consejo Territorial o con la AGE (Guillén y Vilá 2007). En este sentido hay que observar que el régimen competencial adoptado, que es "compartido", ha supuesto la presencia institucional de la Administración Central (a través de la financiación del nivel mínimo y acordado) en los sistemas de servicios sociales autonómicos superando la precaria presencia que suponía el Plan Concertado de Prestaciones Sociales Básicas.

En lo que respecta a las organizaciones de acción social del ámbito de la discapacidad han sido uno de los actores sociales que han salido más reforzados con la ley en lo cual ha influido de manera sustancial su tradicional capacidad de influencia y su significativa capacidad operativa en la red de servicios sociales. Esto les ha servido para posicionarse como un sector a tener en cuenta en el diseño legislativo, consiguiendo la introducción de cambios no determinantes pero significativos en el proyecto de ley, 
que han afectado no solo a su denominación sino también a la valoración "especial" del Tercer Sector (Art.16.2).

En cuanto a la patronal, participó activamente en la no inclusión de la ley en el ámbito de la Seguridad Social. También consiguió cierta atenuación de la iniciativa pública y de la economía social durante el recorrido de preparación y lectura legislativa, así como en los posteriores desarrollos legislativos ${ }^{6}$, haciendo más visibles ciertos productos de financiación privados (por ejemplo, la hipoteca inversa). No obstante, el peso de la iniciativa privada mercantil en el conjunto de la ley sigue siendo poco significativo sobre todo si se compara con las expectativas generadas en el sector, a pesar de lo cual, ello no contradice la realidad de un modelo de servicios sociales en el que la provisión mercantil gana espacio de manera creciente siguiendo la estela de los servicios sociales en el modelo social europeo.

Sobre la LAPAD planean algunos retos e incertidumbres sobre el acceso, la calidad y la sostenibilidad del sistema a nivel interterritorial. Ciertamente, estas incertidumbres, pueden ser atribuibles en algún modo a la complejidad competencial y juventud de la reforma. No obstante, hasta que el SAAD sea evaluado formalmente en 2010 , ya ha sido analizado desde diferentes ámbitos como la Agencia Estatal de Evaluación de las Políticas Públicas y la Calidad de los Servicios (AEVAL 2008), el Grupo de expertos (2009) para la evaluación del desarrollo y efectiva aplicación de la Ley o desde la Asociación de Directores y Gerentes de servicios sociales que emite dictámenes regulares sobre el desarrollo del SAAD y todos ellos ponen de manifiesto las incertidumbres mencionadas en estas páginas.

Aunque la intensidad protectora dependerá del rumbo que finalmente tomen las correcciones que se pretenden introducir en el sistema, se podrían sugerir diversas mejoras. En primer lugar, potenciar la incorporación y potenciación de trabajadores sociales y de los gestores de caso como personas de referencia para coordinar las necesidades de atención de las personas dependientes a nivel municipal. En segundo lugar, reforzar el papel de los ayuntamientos en la gestión de servicios tal y como se contempla en el artículo 12 de la LAPAD, escasamente desarrollado normativamente por las CCAA. Del mismo modo convendría mejorar los sistemas de información sobre la población beneficiaria y el tipo de prestaciones que reciben, un protagonismo más activo de la Administración General del Estado y de los Consejos Consultivos en la coordinación del sistema y mayor implicación de las administraciones sanitarias y de la Seguridad Social, esta última con históricas competencias en este ámbito.

${ }^{6}$ Ley 41/2007, de 7 de diciembre, por la que se modifica la Ley 2/1981, de 25 de marzo, de Regulación del Mercado Hipotecario y otras normas del sistema hipotecario y financiero, de regulación de las hipotecas inversas y el seguro de dependencia y por la que se establecen determinadas normas tributarias. 


\section{REFERENCIAS BIBLIOGRÁFICAS}

Agencia Estatal de Evaluación de las Políticas Públicas y la Calidad de los Servicios (AEVAL). 2008. "La participación de la Administración General del Estado en el Sistema para la Autonomía y Atención a la Dependencia". Madrid: AEVAL. Consultado 30 junio 2009 (http://www.aeval.es).

Asociación Estatal de Directoras y Gerentes en Servicios Sociales (AEDGSS). 2009. "Desarrollo e implantación territorial de la Ley de Promoción de la Autonomía Personal y Atención a las Personas en Situación de Dependencia, III Dictamen". Madrid: AEDGSS. Consultado 5 de junio 2009 (http:// www.directoressociales.com/).

Casado, D. 2004. Respuestas a la Dependencia. Madrid: CCS.

CERMI (Comité Español de Representantes de Personas con Discapacidad). 2005. "Posición del movimiento asociativo articulado de la Discapacidad ante la futura regulación legal de la promoción de la autonomía personal y la atención a las situaciones de dependencia". Madrid: CERMI. Consultado 20 mayo 2009 (http://www.cermi.es).

CES (Consejo Económico y Social). 2006. "Dictamen 3/2006, sobre el Anteproyecto de Ley de Promoción de la Autonomía Personal y Atención a las Personas en Situación de Dependencia". Madrid: CES. Consultado 3 mayo 2009 (http://www.ces.es).

Consejo de Estado. 2006. "Dictamen del Consejo de Estado sobre el Anteproyecto de Ley de Promoción de la Autonomía Personal y Atención a las Personas en Situación de Dependencia". Madrid: Consejo de Estado. Consultado 5 mayo 2009 (http://www.seg-social.es/imserso/dependencial dictamenconest.pdf).

Centro de Investigaciones Sociológicas (CIS). 2004. "Estudio n. ${ }^{0}$ 2581, Barómetro de noviembre de 2004". Madrid: Centro de Investigaciones Sociológicas. Consultado 20 abril 2009 (http://www.cis. es/cis/opencm/ES/1_encuestas/estudios/ver.jsp?estudio=4355).

Defensor del Pueblo. 2000. La atención sociosanitaria en España: perspectiva gerontológica y otros aspectos conexos. Madrid: Defensor del Pueblo.

European Commission. 2009. Joint Report on Social Protection and Social Inclusion 2009. Luxembourg: European Commission.

Grupo de Expertos. 2009. Informe final del grupo de expertos para la evaluación del desarrollo y efectiva aplicación de la Ley 39/2006, de 14 de diciembre de Promoción de la autonomía personal y Atención a las situaciones de dependencia. Madrid: MSYPS-IMSERSO.

Guillén, E. 2005. Sobre problemas y respuestas sociales. Barcelona: Hacer.

Guillén, E. y A. Vilà. 2007. "Impacto de la Ley de Promoción de la Autonomía Personal y Atención a las Personas en Situación de Dependencia en los servicios sociales de las Comunidades Autónomas". Pp 177-208 en Perfeccionamiento de los servicios sociales en España: informe con ocasión de la ley sobre autonomía y dependencia. Madrid: Caritas.

IMSERSO. 2000. Las personas mayores en España. Informe 2000. Madrid: Ministerio de Trabajo y Asuntos Sociales. 
IMSERSO (Instituto de Mayores y Servicios Sociales). 2002. Las personas mayores en España. Informe 2002. Madrid: Ministerio de Trabajo y Asuntos Sociales.

IMSERSO (Instituto de Mayores y Servicios Sociales). 2004. Las personas mayores en España. Informe 2004. Madrid: Ministerio de Trabajo y Asuntos Sociales.

IMSERSO (Instituto de Mayores y Servicios Sociales). 2005. Libro Blanco de atención a las personas dependientes en situación de dependencia en España. Imserso. Madrid: Ministerio de Trabajo y Asuntos Sociales. Consultado 20 marzo 2009 (http://www.imsersomayores.csic.es/documentos/ documentos/libroblancodependencia/mtas-libroblancodependencia-01.pdf).

IMSERSO (Instituto de Mayores y Servicios Sociales). 2006. Las personas mayores en España. Informe 2006. Madrid: Ministerio de Trabajo y Asuntos Sociales.

IMSERSO (Instituto de Mayores y Servicios Sociales). 2009. Las personas mayores en España. Informe 2008. Madrid: Ministerio de Trabajo y Asuntos Sociales.

España. 2006. "Ley 39/2006, de 14 de diciembre, de Promoción de la Autonomía Personal y Atención a las personas en situación de dependencia". Boletín Oficial del Estado 299: 44142-44156, 8 abril 2009.

Marbán, V. 2006. “Tercer Sector y Dependencia”. Documentación Social 141: 145-162.

Marbán, V. 2009. "La atención a la Dependencia" Pp. 207-238 en Moreno. L. Reformas de las políticas del bienestar en España. Madrid: Ed. Siglo XXI.

Montserrat, J. 2009. "Evolución y perspectivas de la financiación del sistema para la autonomía y atención a la dependencia". Documentación Administrativa 276/277: 411-442.

Montserrat, J. 2007. "El impacto de la ley de la dependencia y del efecto demográfico en los gastos de los cuidados de larga duración". Estudios de Economía Aplicada 25: 379-406.

Moreno, L. 2009. Reformas de las políticas del bienestar en España. Madrid: Ed. Siglo XXI.

Pacolet, J. 1998. Social Protection for Dependency in Old Age in the 15 EU Member Status and Norway. Brussels: European Commission.

Pacolet, J. 2006. "Protección social de las personas mayores dependientes: sostenibilidad del Estado del Bienestar y ámbito del seguro de cuidados de larga duración." Revista Española del Tercer Sector 3:111-160.

Pavolini, E. y C. Ranci. 2008. "Restructuring the Welfare State: reforms in long-term care in Western European countries". Journal of European Social Policy 18: 246-259.

Rodríguez Cabrero, G. 2004. "Protección social de la dependencia en España". Documento de trabajo 44/2004. Fundación Alternativas: Madrid.

Rodríguez Cabrero, G. 2005. "Los retos del Tercer Sector en España en el espacio social europeo. Especial referencia a las organizaciones de acción social". Revista Española del Tercer Sector 1: 63-91. 
Rodríguez Cabrero, G. 2009. "El desarrollo de la política social de promoción de la autonomía y atención a las personas en situación de dependencia en España (2007-2009)". Gestión y Análisis de Políticas Públicas 2: 33-58.

Rodríguez Rodríguez, P. 2006. "El sistema de servicios sociales español y las necesidades derivadas de la atención a la dependencia". Documento de trabajo 87/2006. Fundación Alternativas: Madrid.

Sancho, M y Rodríguez, P. 2002. "Envejecimiento y protección social de la dependencia en España. Veinte años de historia y una mirada hacia el futuro". Revista de Intervención Psicosocial 3: 259-75.

Sabatier, P. A. y H. Jenkins-Smith. 1999. "The Advocacy Coalition Framework: an assessment". Pp. 117-168 in Sabatier, P.A. 1999. Theories of the Policy Process. Boulder, Co: Westview Press.

SEGG (Sociedad Española de Geriatría y Gerontología) 2006. "Posicionamiento de la SEGG sobre el Anteproyecto de Ley de Promoción de la Autonomía Personal y Atención a las Personas en Situación de Dependencia". Madrid: SEGG. Consultado 4 abril 2009 (http://www.imsersomayores. csic.es/documentos/productos/dependencia/segg-posicionamiento-01.pdf).

SIPOSO (Seminario de Intervención y Políticas Sociales). 2006. "Informe sobre el Anteproyecto de Ley de Promoción de la Autonomía Personal y Atención a las Personas en Situación de Dependencia". Madrid: SIPOSO. Consultado 4 abril 2009 (http://www.polibea.com/siposo/Informe24-2-06.pdf).

VICENTE MARBÁN GALLEGO es Profesor contratado doctor en la Universidad de Alcalá, Director Adjunto de la Revista Española del Tercer Sector y miembro del Consejo Asesor de diversas revistas de índole académica. Investigador en diversas investigaciones europeas y nacionales y autor de diversos libros y artículos, entre ellos en revistas como Civil Szemle, 2009/3, Política y Sociedad, Vol 44, $n^{0} 2$; Revista CIRIEC, $n^{\circ} 56$, o Revista Internacional de Sociología, $n^{\circ} 30$.

RECIBIDO: $29 / 06 / 2010$

ACEPTADO: $15 / 03 / 2011$ 